\title{
NOTCH SIGNALING PREVENTS MUCOUS METAPLASIA IN MOUSE CONDUCTING AIRWAYS DURING POSTNATAL DEVELOPMENT
}

\author{
P.-N. Tsao ${ }^{1}$, S.-C. Wei ${ }^{2}$, M.-F. Wu ${ }^{3}$, M.-T. Huang ${ }^{4}$, H.-Y. Lin ${ }^{5}$, M.-C. Lee ${ }^{2}$, K.M.-C. Lin ${ }^{6}$, I.-J. Wang ${ }^{7}$, V.

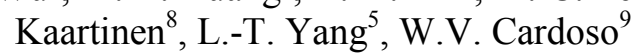 \\ ${ }^{1}$ Pediatrics, ${ }^{2}$ Internal Medicine, National Taiwan University Hospital, ${ }^{3}$ Animal Medical Center, College of \\ Medicine, National Taiwan University, ${ }^{4}$ Medical Research, National Taiwan University Hospital, Taipei, \\ ${ }_{5}^{5}$ Institute of Cellular and Systems Medicine, ${ }^{6}$ Division of Medical Engineering, National Health Research \\ Institutes, Miaoli County, ' Ophthalmology, National Taiwan University Hospital, Taipei, Taiwan R.O.C., \\ ${ }^{8}$ Biologic and Materials Sciences, University of Michigan School of Dentistry, Ann Arbor, MI, ${ }^{9}$ Pulmonary \\ Center, Boston University School of Medicine, Bonton, MA, USA
}

Background and aims: Goblet cell metaplasia and mucus overproduction contribute to the pathogenesis of chronic lung diseases, including asthma, chronic obstructive pulmonary disease. Notch signaling regulates cell fate decisions and plays a critical role in goblet cell differentiation in the gut epithelium. However it is unclear whether endogenous Notch influences goblet cell differentiation in airways.

Methods: We inactivated Notch signaling conditionally in the lung using a Tgfb3-Cre deleter mouse line, which targets the airway epithelium in a mosaic fashion, and mice carrying floxed alleles of the Pofut 1 gene, which encodes an O-fucosyltransferase essential for Notch-ligand binding. Immunohistochemistry, cell culture and transfection were used to analyze the lung phenotype and possible mechanism.

Results: Mice were viable but showed an aberrant airway phenotype with decreased Clara cell number and marked goblet cell metaplasia during postnatal life. The phenotype was confirmed by a panel of goblet cell markers, showed no changes in cell proliferation or altered expression of proinflammatory cytokines and was associated with significant downregulation of Hes5. Luciferase reporter analysis suggested that Notch directly repressed MUC5AC transcription in lung epithelial cells. The same phenotype in mice in which the Notch transcriptional effector Rbpjk was deleted indicated the involvement of the canonical Notch pathway.

Conclusions: The data suggested that during postnatal life Notch signaling is required to maintain the Clara cell phenotype and prevent goblet cell differentiation in proximal airways. Notch may be critical in regulating the response of the lung to environmental injurants or allergens that result in goblet cell metaplasia. 